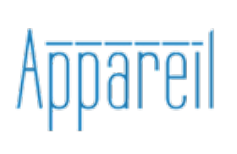

Appareil

14 | 2014

Esthétiques latino-américaines : penser à rebours

\title{
Le cinéma ou l'art de laisser revenir les fantômes : une approche à partir de J. Derrida
}

Adolfo Vera

\section{(2) OpenEdition}

1 Journals

\section{Édition électronique}

URL : http://journals.openedition.org/appareil/2115

DOI : 10.4000/appareil.2115

ISSN : 2101-0714

Éditeur

MSH Paris Nord

Référence électronique

Adolfo Vera, "Le cinéma ou l'art de laisser revenir les fantômes : une approche à partir de J. Derrida », Appareil [En ligne], 14 | 2014, mis en ligne le 12 décembre 2014, consulté le 30 juillet 2020. URL : http:// journals.openedition.org/appareil/2115; DOI : https://doi.org/10.4000/appareil.2115

Ce document a été généré automatiquement le 30 juillet 2020

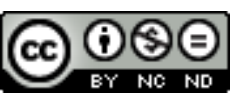

Appareil est mis à disposition selon les termes de la Licence Creative Commons Attribution - Pas d'Utilisation Commerciale - Pas de Modification 4.0 International. 


\title{
Le cinéma ou l'art de laisser revenir les fantômes : une approche à partir de J. Derrida
}

\author{
Adolfo Vera
}

1 On pourrait appliquer à une théorie politique du cinéma les acquis derridiens en ce qui concerne les rapports entre trace et spectralité. L'image est une trace : cela veut dire qu'elle est une inscription et qu'elle suppose donc une surface d'inscription qui est nécessairement technique. À partir de Derrida, nous observons qu'à la base de toute surface d'inscription il y a la répétition, le fort-da, et donc la pulsion de mort qui est aussi pulsion de hantise et de revenance : la temporalité propre à cette configuration est donc celle de la Nachträglichkeit. La question du document, et d'une image-document, et de ce fait de l'archive, est de cette façon posée.

2 Comment la définition d'une image en mouvement, et donc d'une trace en mouvement, affecterait-elle la définition de la spectralité (en tant que puissance de revenance)? Comment cette définition impliquerait-t-elle aussi une redéfinition du témoignage? Dans un entretien à l'occasion des cinquante ans des Cahiers du cinéma ${ }^{1}$, Derrida revient sur son expérience (non-cinéphile, comme il l'avoue) du cinéma. La question de la spectralité est pour lui la plus significative. Elle est posée dans les termes suivants :

L'expérience cinématographique appartient, de part en part, à la spectralité, que je relie à tout ce qu'on a pu dire du spectre en psychanalyse - ou à la nature même de la trace. Le spectre, ni vivant ni mort, est au centre de certains de mes écrits, et c'est en cela que, pour moi, une pensée du cinéma serait peut-être possible [...] Le cinéma peut mettre en scène la fantomalité presque frontalement, certes, comme une tradition du cinéma fantastique, les films de vampires ou de revenants, certaines œuvres d'Hitchcock. Il faut distinguer cela de la structure de part en part spectrale de l'image cinématographique. Tout spectateur, lors d'une séance, se met en communication avec un travail de l'inconscient qui, par définition, peut être rapproché du travail de la hantise selon Freud. Il appelle cela ce qui est «étrangement familier » (unheimlich) $[. ..] »^{2}$. 
Derrida continue son argumentation en insistant sur les rapports profonds qui lient le cinéma et la psychanalyse (Benjamin étant le premier à l'avoir compris) :

Même la vision et la perception du détail dans un film sont en relation directe avec le procédé psychanalytique. L'agrandissement n'agrandit pas seulement, le détail donne accès à une autre scène, une scène hétérogène. La perception cinématographique n'a pas d'équivalent, mais elle est la seule à pouvoir faire comprendre par l'expérience ce qu'est une pratique psychanalytique: hypnose, fascination, identification, tous ces termes et ces procédés sont communs au cinéma et à la psychanalyse, et c'est là le signe d'un "penser ensemble » qui me semble primordial. $^{3}$

On sait bien que quand Derrida parle de «fantôme » en psychanalyse, il parle de Torok et Abraham et de leur «clinique du fantôme ». En même temps, pour lui le cinéma a instauré un nouveau (et inouï) régime de croyance. Quand on est dans une salle de cinéma, on «croit » à ce que l'on voit projeté sur l'écran, et cela du fait que l'on est dans un état où notre conscience n'exerce pas ses droits critiques. Et cela concerne le caractère hypnotique du cinéma, et les effets politiques (la question de la propagande) que cela implique. D'autre part, croire aux spectres n'est pas quelque chose de «normal ». «Puisque la dimension spectrale, continue Derrida, n'est celle ni du vivant, ni celle du mort, ni celle de l'hallucination ni celle de la perception, la modalité du croire qui s'y rapporte doit être analysée d'une façon absolument originale $»^{4}$. Or, ce qui se produit lors de cette projection particulière qu'est le cinéma, est une "greffe » de spectralité, c'est-à-dire, l'inscription sur la pellicule projetée des " traces de fantômes " qui, en tant que traces, s'inscrivent et se désinscrivent en obéissant à la loi de la revenance et de la hantise, en véhiculant de cette façon les «deuils» de l'histoire. «Mémoire spectrale, le cinéma est un deuil magnifique, un travail du deuil magnifié. Et il est prêt à se laisser impressionner par toutes les mémoires endeuillées, c'est-à-dire par les moments tragiques et épiques de l'histoire $»^{5}$.

Or, si le cinéma définit un "nouveau régime de la croyance », il doit aussi définir un nouveau régime du témoignage. On croit, selon Derrida, au témoignage, et c'est à cause de cela qu'il se rapproche de l'acte de la promesse ${ }^{6}$. Cela n'empêche pas que, du fait de l'image cinématographique, cette croyance qui communique avec le témoignage soit par définition objet de la méfiance, juridique, par exemple. C'est la raison pour laquelle Derrida s'intéresse, dans cet entretien, à Shoah de Claude Lanzman. Les rapports entre témoignage, spectralité et image y sont établis comme dans aucun autre film. L'image est vouée à la survivance des fantômes, et cela du fait de sa capacité inscriptive, et donc technique, particulière. Dans ce sens, le film de Lanzmann serait fidèle au plus haut degré aux possibilités techniques propres à l'appareil et qui impliquent le témoignage. La trace est, en tant que concept qui réfère à une existence concrète inscrite sur la pellicule puis projetée sur l'écran, le pilier théorique de l'analyse.

La trace, explique Derrida, est le «ça a eu lieu là » du film, la survivance. Car tous ces témoins sont des survivants : ils ont vécu ça et le disent. Le cinéma est le simulacre absolu de la survivance absolue. Il nous raconte ce dont on ne revient pas, il nous raconte la mort. Par son propre miracle spectral, il nous désigne ce qui ne devrait pas laisser de trace. Il est donc deux fois trace : trace du témoignage luimême, trace de l'oubli, trace de la mort absolue, trace du sans trace, trace de l'extermination. C'est le sauvetage, par le film, de ce qui reste sans salut, l'expérience de la survivance pure qui témoigne. ${ }^{7}$

6 La trace donc comme "survivance " qui témoigne d'elle-même. D'autre part, Derrida pointe le fait que ce témoignage-là, en tant qu'il est cinématographique, se structure à 
partir d'aspects spécifiques de la "réalité matérielle », notamment les gestes du corps et de la voix. Il nous faudra donc dire que s'il y a de la survivance cinématographique, et de ce fait de la spectralité et une possibilité de témoigner, c'est comme conséquence des spécificités techniques propres à l'appareil, celles de l'enregistrement du «monde d'en bas » (Kracauer) d'abord, mais aussi celles du montage, et de la projection lors de la « séance », avec toutes les conséquences de croyance que cela implique.

7 Pour Derrida donc, l'image en général, en tant que trace, est un type d'inscription qui implique une désinscription (comme une signature implique une contresignature), et c'est dans ce décalage, dans cet espace ouvert entre inscription et désinscription, que s'introduit la spectralité et sa puissance de destruction des catégories préétablies. C'est la question de l'appropriation-réappropriation qui est donc ainsi mise en jeu. Dans un dialogue avec des théoriciens de l'image au Collège iconique de l'Institut national d'histoire de l'art, en juin 2002, Derrida élaborait cette problématique de la façon suivante :

[...] la circoncision, la trace, la coupure comme interruption de l'appropriation. Coupure d'avec soi qui est la condition de cette expérience, non seulement grâce à la circoncision au sens littéral, mais à ce qui est dit à un moment donné d'une circoncision qui n'est pas seulement propre à la culture juive ou à telle ou telle culture ou religion abrahamique, juive ou musulmane, mais propre à une expérience universelle qui suppose cette coupure, cette non réappropriabilité de l'idiome. Ce qui est absolument singulier chez chacun de nous, ce qui est absolument idiomatique, la signature disons, c'est paradoxalement ce qui ne peux pas me réapproprier. Ça m'est absolument propre, mais je ne peux pas me le réapproprier, c'est ça le paradoxe, et c'est ce qu'un film nous donne à penser. ${ }^{8}$

Derrida dit ces phrases en parlant de sa propre expérience cinématographique, d'abord en tant que comédien dans le film Ghost Dance (1983) de Ken McMullen et puis comme «objet » des documentaires D'ailleurs Derrida (1999) de Safaa Fathy et Derrida (2002) de Kirby Dick, en se référant donc à l'expérience personnelle de se voir projeté, dédoublé, sur l'écran. Cependant, il s'agit d'une expérience universelle: les sujets que nous regardons sur l'écran ne sont que des traces cinématographiques, et donc des "doubles", des "fantômes", même si nous pouvons être certains qu'il s'agit de ces sujets-là. Derrida insiste sur le fait que, pour que cette "vérité" de la trace cinématographique (mais, pourrions-nous ajouter, de toute trace) s'accomplisse en toute profondeur, le « sujet » sur l'écran doit être, de préférence, quelqu'un qui est déjà mort. À ce moment-là on est face, sans aucun doute et sans métaphore, à un fantôme. C'est bien dans ce sens que Derrida signale, en pensant à son expérience dans le film de Safaa Fathy :

Ça part de moi, c'est-à-dire que ça procède de moi et que, procédant de moi, ça se sépare de moi. C'est pour ça que ça laisse une trace. Moi je peux mourir en chaque instant, la trace reste là. La coupure est là. C'est une part de moi qui est coupée et qui donc part du moi au deux sens du terme : elle procède, elle émane de moi mais en même temps en se séparant, en se coupant, en se détachant de moi [...]."

Cette conception, qui coïncide étonnamment avec la conception «spirite » que Balzac se fait de la photographie ${ }^{10}$, met l'accent sur le fait que toute trace possède, en raison de sa structure, un moment de séparation, de détachement et d'effacement qui est aussi important que celui de l'inscription. Et c'est pour cela que la définition de la trace ne coïncide pas avec celle de l'index. Derrida parlera donc de la « restance» de la trace, une restance "au-delà de toute ontologie ${ }^{11}$. Or, cette définition de la trace devra nécessairement affecter celle de l'archive. Une archive n'est autre chose qu'une 
« sélection de traces », et de ce fait elle-même, en tant qu'archive, se fonde en effaçant certaines traces et en en conservant d'autres. Cette dialectique conservation/ effacement est une dialectique spectrale ${ }^{12}$. C'est, justement, le «mal d'archive » : celleci n'est pas un objet de mémoire, au contraire elle est par essence plus proche de l'oubli.

10 C'est à partir de cela qu'on peut vérifier l'importance d'un film comme Ghost Dance (1983) de Ken McMullen. Au-delà des « apparitions » - conservons à ce terme toutes ses significations, y compris spectrales - de Derrida, la question politique est posée d'emblée, mais aussi celle des nouvelles technologies de l'image. Le nœud du récit s'articule autour de l'expérience de deux jeunes femmes, qui changent constamment de personnalité et sont possédées par diverses figures historiques et anthropologiques (clochards-chiffonniers des villes postmodernes, anciens Indiens d'Amérique, combattants de la Commune de Paris, etc.), qui appartiennent toutes à la catégorie de ce qu'après Benjamin on appelle les «vaincus de l'histoire ». Il s'agit de la dérive sans but de deux jeunes femmes dans les espaces vides ou peuplés de deux grandes métropoles comme peuvent l'être Paris et Londres. En ce sens, le film est une réflexion autour de la survivance des vaincus de l'histoire dans les villes post-modernes. MacMullen insiste systématiquement, pour créer ces "effets de spectralité ", sur la juxtaposition de voix off différentes, qui se superposent aux images, soit d'endroits abandonnés et oubliés des villes (par exemple à Londres, le tombeau de Marx ou les cours vides d'une usine abandonnée, à Paris le mur des fusillés de la Commune, au Père Lachaise), soit de "non-lieux» industriels délaissés, propices à l'apparition de fantômes. Et les voix parlent de rituels, d'électricité, de mythologies, toujours avec la musique de chants indiens, tandis que l'on observe les images d'énormes métropoles, la nuit, traversées par les lumières de voitures et de routes. Ces voix disent, par exemple :

[voix d'un homme] Dans un âge de ténèbres, en un âge et un temps reculés, lors de périodes de deuil, les vivants attaquaient les morts, leur lançant pierres et insultes, crachant et hurlant de rage, car ils se sentaient abandonnés aux terreurs de la nuit... [voix d'une femme] Pourquoi ne puis-je pas oublier? C'est comme si j'étais responsable. Pourquoi je me réveille dans la nuit avec le sentiment qu'ils me regardent? Merde, j'ai si peur. Ils sont tous là à parler de moi. Bon dieu, j'ai besoin de silence. [...] [voix d'un autre homme] On pensait que les fantômes seraient oubliés en cette ère électronique. Mais ils se mirent à utiliser les gadgets électroniques à leurs fins. Ils sautent souvent sur les ondes radios. On a signalé des cas de fantômes dans des boutiques d'électroménager.

11 Et la question de la marchandise est donc ainsi posée. Juste après ces dernières phrases, l'une des jeunes femmes rentre dans une boutique d'électroménager pour vendre des engins électriques (téléphone, machine à écrire électrique, radio, etc.). Elle se fait agresser violemment par le vendeur, qui avant de détruire ses objets, lui dit (en mangeant le téléphone, allusion au cannibalisme d'incorporation si important pour la théorie d'Abraham et Torok) : «Ici, quand c'est moi qui les vends, ça marche, quand c'est toi, ça vaut rien ». Et nous savons combien de pages a dédié Derrida, notamment dans Spectres de Marx, à la définition spectrale de la valeur marchande.

Ghost Dance est donc un film sur la hantise et sur la revenance. À un autre moment du film, une voix féminine dit :

Brusquement, « je » et « moi » sont devenus deux personnes distinctes. On marchait dans une ville. La nuit tombait, le ciel rayonnait de lumières électriques. On s'est mis à marcher vers l'océan. Soudain, les gens se sont mis à courir, en sens opposé. C'était la panique. Mais ceux qui se précipitaient vers nous étaient les morts des 
siècles passés. Leur poids écrasant se transforma en vague colossale. Elle nous engloutit. Et une seule d'entre nous survécut.

Il s'agit d'un film dans lequel ce que Lyotard appelle les « normes de discours » (norme primitive de la narration, norme théologique de la révélation, norme moderne de la délibération ${ }^{13}$ ) se superposent et interagissent, comme si, du fait de l'appareil cinématographique, le discours philosophique autour de la spectralité de Derrida pouvait se juxtaposer aux références à la narration primitive et aux rites d'invocation des morts, le tout dans un contexte urbain postmoderne et capitaliste.

Les phrases dites par Derrida lors de ses « apparitions » insistent sur la question de la revenance et la hantise qui est d'une grande importance pour toute philosophie intéressée aux effets esthétiques et politiques d'une théorie de la spectralité comme celle proposée par Derrida. La revenance et la hantise sont avant tout des modes de la temporalité - destruction de la temporalité que la métaphysique de la présence a imposée à l'histoire, soit comme théorie du progrès, soit comme prédominance de la mémoire, etc. Revenance et hantise sont le moyen par lequel la spectralité écrase le présent et toute manifestation de la "présence ». Cela implique que nous ne pouvons plus être des contemporains ${ }^{14}$ dans le sens de la co-présence, mais seulement dans l'anachronisme et la simultanéité des temporalités divergentes qui s'interspectralisent justement parce qu'elles se "hantent » mutuellement, en se trouvant en plus dans l'obligation d'assumer la revenance des disparus et des vaincus, comme les voix de ces femmes et de ces hommes dans le film de MacMullen. "Être hanté par un fantôme, dit Derrida dans le film, c'est avoir la mémoire de ce qu'on n'a jamais vécu au présent, avoir la mémoire de ce qui, au fond, n'a jamais eu la forme de la présence ». Et, à la question de l'une des jeunes femmes: "Est-ce que vous croyez aux fantômes?», Derrida répond par une autre question : «Est-ce qu'on demande à un fantôme s'il croît aux fantômes?». Et puisque il est conscient qu'une trace cinématographique n'est qu'un fantôme, Derrida ajoute : «Ici le fantôme c'est moi ». Et il continue : « Le cinéma c'est l'art de laisser revenir les fantômes $»^{15}$.

\section{BIBLIOGRAPHIE}

Agamben Giorgio, Qu'est-ce que le contemporain?, Paris, Rivages, 2008.

De Baecque Antoine, Jouisse Thierry, « Jacques Derrida. Le cinéma et ses fantômes », Cahiers du cinéma, $\mathrm{n}^{\circ}$ 50, avril, 2001.

Derrida Jacques, « Trace, archive, image et art », dialogue au Collège iconique (avec Jean-Michel Rodes, François Soulages, Gérald Cahen, Patrick Charadeau, Gérard Hubert, Serge Tisseron et Marie-José Mondzain), Derrida en castellano, le 25/06/2002, en ligne : http://

www.jacquesderrida.com.ar/frances/trace_archive.htm

Derrida Jacques, Poétique et politique du témoignage, Paris, L'Herne, 2005.

Derrida Jacques, Bernard Stiegler, Échographies de la télévision, Paris, Galilée-INA, 1996.

Krauss Rosalind, Le photographique. Pour une théorie des écarts, Paris, éditions Macula. 
Lyotard Jean-François, Le différend, Paris, Minuit, 1983.

\section{NOTES}

1. Antoine De Baecque, Thierry Jouisse, «Jacques Derrida. Le cinéma et ses fantômes ", Cahiers du cinéma, $\mathrm{n}^{\circ}$ 50, avril, 2001, p. 75-85.

2. Ibid., p. 77. C'est moi qui souligne.

3. Ibid.

4. Ibid. p. 78.

5. Ibid.

6. Jacques Derrida, Poétique et politique du témoignage, Paris, L'Herne, 2005.

7. Ibid., p. 80.

8. Jacques, Derrida, "Trace, archive, image et art ", dialogue au Collège iconique (avec JeanMichel Rodes, François Soulages, Gérald Cahen, Patrick Charadeau, Gérard Hubert, Serge Tisseron et Marie-José Mondzain), Derrida en castellano, le 25/06/2002, en ligne: http:// www.jacquesderrida.com.ar/frances/trace_archive.htm

9. Ibid.

10. Rosalind Krauss, "Sur les traces de Nadar ", in Le photographique. Pour une théorie des écarts, Paris, Macula, p. 18-36.

11. Jacques Derrida, «Trace, archive, image et art », Ibid.

12. « Là où l'on garderait tout, il n'y aurait pas d'archives. L'archive commence par la sélection, et cette sélection est une violence [...] Donc l'archive commence là où la trace s'organise, se sélectionne, ce qui suppose que la trace est toujours finie. Qu'est-ce que ça veut dire? Ça veut dire qu'une trace peut toujours s'effacer ». Ibid.

13. Jean-François Lyotard, Le différend, Paris, Minuit, 1983.

14. Giorgio Agamben, Qu'est-ce que le contemporain?, Paris, Rivages, 2008.

15. Derrida lui-même a signalé lors d'un dialogue avec Bernard Stiegler à quel point la mort de la comédienne Pascale Ogier, intervenue quelque temps après le tournage du film Ghost Dance, l'avait impressionné sur le plan de la spectralité : «À la fin de mon improvisation, je devais lui dire : et vous alors, est-ce que vous y croyez, aux fantômes? Et en la répétant au moins trente fois, à la demande du cinéaste, elle dit cette petite phrase : “Oui, maintenant, oui”. Déjà dans la prise de vues, elle l'a répétée au moins trente fois. Déjà ce fut un peu étrange, spectral, décalé, hors de soi, cela arrivait plusieurs fois en une fois. Mais imaginez quelle a pu être mon expérience quand, deux ou trois ans après, alors que Pascale Ogier, dans l'intervalle, était morte, j'ai revu le film aux États-Unis [...] J'ai vu tout à coup arriver sur l'écran le visage de Pascale, que je savais être le visage d'une morte. Elle répondait à ma question: "Croyez-vous aux fantômes?" En me regardant quasiment dans les yeux, elle me disait encore, sur grand écran : "Oui, maintenant, oui”. Quel maintenant? Des années après au Texas, j'ai pu avoir le sentiment bouleversant du retour de son spectre, le spectre de son spectre revenant me dire, à moi ici, maintenant: "Maintenant... maintenant... maintenant, c'est-à-dire dans cette salle obscure d'un autre continent, dans un autre monde, là, maintenant, oui, crois-moi, je crois aux fantômes" ». Jacques Derrida, Bernard Stiegler, Échographies de la télévision, Paris, Galilée-INA, 1996, p. 133-135. 


\section{RÉSUMÉS}

Spectres de Marx (1993) aura été l'ouvrage qui aura donné ses lettres de citoyenneté philosophique à la catégorie de spectralité en tant qu'elle est en mesure de rendre compte de complexes rapports entre violence politique, crise de la représentation et nouvelles technologies. Nous essayerons dans cet article de pointer comment cette théorie pourrait aussi porter ses fruits en considérant le cas de l'appareil cinématographique. Pour ce faire, on considérera notamment deux interventions publiques - Derrida n'a presque pas écrit sur le cinéma - où Derrida a mis au point ce que nous pourrions appeler un programme théorique pour établir une philosophie spectrale du cinéma, où les questions - essentielles dans sa pensée - de la trace, de l'archive, de l'oubli, de la répétition et de la technique seront fondamentales.

INDEX

Mots-clés : trace, spectralité, archive, répétition, technique

\section{AUTEUR}

\section{ADOLFO VERA}

Professeur Institut de philosophie, Université de Valparaíso, adolfovera27@gmail.com 